\title{
Origen, evolución y realidades de las empresas públicas universitarias en el Ecuador
}

\section{Origin, evolution and realities of public university companies in Ecuador}

John Alexander Campuzano Vásquez

Patricia Alexandra Uriguen Aguirre

Gonzalo Junior Chávez Cruz

Holger Fabrizzio Bejarano Copo

Víctor Betancourt Gonzaga

\section{Universidad Técnica de Machala, Ecuador}

Autor para correspondencia: jcampuzano@utmachala.edu.ec,puriguen@utmachala.edu.ec, gchavez@utmachala.edu.ec, hberajano@utmachala.edu.ec

Fecha de recepción: 20 de julio de 2018 - Fecha de aceptación: 30 de agosto de 2018

Resumen: El presente artículo realiza una revisión extensiva del estado legal de las empresas públicas universitarias en el Ecuador, para ello examina la fundamentación teórica, legal, educativa y económica que permite la creación de empresas públicas universitarias en las instituciones de educación superior públicas y de los diferentes servicios y/o productos que ofrecen. Mediante revisión documental de la ley orgánica de educación superior, de empresas públicas y de normativas internas se procedió a desarrollar un análisis documental que recoge la evolución temporal de estas empresas, para luego establecer un marco comparativo actualizado de las empresas existentes que registre particularidades entre ellas a partir del objeto social de su creación. Se encontró que muchas empresas públicas tienen diferentes órganos de control que responden a las diferentes realidades universitarias, así como que son creadas como una vía para crear recursos adicionales en las universidades ante los permanentes problemas de financiamiento presupuestario por parte del gobierno central, en la mayoría de los casos analizados no hay una verdadera vinculación del conocimiento creado en institutos de investigación, departamentos o de académicos que son parte de estas instituciones de educación superior con el sector privado, comunitario y público local en las provincias en las que realizan actividades empresariales estas organizaciones productivas universitarias.

Palabras Claves: empresas públicas; educación superior; gobierno; universidades

Abstract: This article carries out an extensive review of the legal status of public university enterprises in Ecuador, for which it examines the theoretical, legal, educational and economic foundation that allows the creation of public university enterprises in public higher education institutions and the different services and / or products that they offer. Through a documentary review of the organic law of higher education, of public companies and internal regulations, a documentary analysis was developed that includes the time evolution of these companies, to then establish an updated comparative framework of existing companies that register particularities among them. from the social object of its creation. It was found that many public companies have different control bodies that respond to the different university realities, as well as being created 
as a way to create additional resources in the universities in the face of the permanent problems of budgetary financing by the central government, in the majority of the cases analyzed, there is no true linkage of the knowledge created in research institutes, departments or academics that are part of these higher education institutions with the private, community and local public sectors in the provinces in which these organizations carry out business activities productive university.

Key Words: public enterprises; higher education; government; universities

\section{Introducción}

La creación de empresas de productos y servicios al interior de las universidades ecuatorianas no ha sido algo muy común desde el siglo pasado, no así en naciones de mayor desarrollo, en las que iniciativas de investigación denominadas spin off demuestran como la labor de investigadores de laboratorios universitarios aportan con resultados y tecnología al sector de la producción (Iglesias, Jambrino, \& Peñafiel, 2012).

En Ecuador las empresas públicas en los últimos ocho años están tratando de promover dinamismo empresarial y descentralización del manejo de recursos, un viejo problema en las instituciones de educación superior (IES en adelante). Tal vez, a esta presencia contribuya la concepción ideológica y económica de creer que tener presencia estatal empresarial, hace del Estado un dirigente activo e importante en dicho sector y en la creación de empleo (MiñoCascante \& Roldan-Ruenes, 2015).

Se piensa entonces que las empresas al nacer de la academia tienen altísimas probabilidades de éxito (Fong, 2010). En este sentido las IES están usando a la vinculación con la sociedad como un medio potente para crear empresas, acercándose o buscando satisfacer necesidades de la sociedad tanto en lo social, societario y comunitario. El modelo de triple hélice de Leydesdorff \& Etzkowitz (1998) sirve de sustento para la creación de empresas públicas, al manifestar la unión entre empresas, gobierno y universidades, lo que denominan triángulo dinámico que se basa en las necesidades de los dos primeros actores.

De acuerdo a Gibson \& Mahdjoubi (2010) citado por Ferreiro (2016) consideran que el papel que juega la transferencia de tecnología al exterior desde las universidades como resultado de las investigaciones, produce nuevas herramientas que se aplican a la producción en las empresas, manifestándose con mucho éxito porque nacen desde la academia.

Hoy en día las IES ya no solo deben estar creando conocimiento para las estanterías, más bien deben crearlo con la posibilidad de transferir tecnología, y un buen mecanismo para ello es la creación de empresas con base tecnológica, de provisión de servicios, o de transformación productiva. Si bien las IES en Ecuador están incursionando en estos nuevos proyectos, presentan algunos problemas en costos, por lo que no todas las empresas creadas desde la universidad consiguen resultados exitosos, esto ha dado lugar a que se limiten en su creación Fong (2006) citado por (Méndez, Hernández, Lino, \& González, 2014).

Muchas universidades de todo el mundo están creando spin-off, nuevas empresas cuyo modelo de gestión es diferente a las creadas en Ecuador que son públicas sin orientación tecnológica, aunque ya se piensa en un acercamiento entre academia y negocio manifestado en la propiedad intelectual de sus descubrimientos (Lopez, 2017). Este acercamiento es compartido 
por Beraza \& Rodriguez (2010) los que añaden, que el desarrollo de una región o un país está en función de las propuestas de las universidades, y para ello hay la necesidad de difundir los resultados de sus investigaciones y buscar una verdadera vinculación con la sociedad.

Con estos antecedentes es relevante en esta investigación describir el origen, evolución y el estado actual de las empresas públicas, de manera que se tenga información actualizada que oriente a directivos de universidades públicas y de control a nivel nacional como el Consejo de Educación Superior sobre el impacto que tienen en la investigación y en la vinculación con la sociedad, o si estas se constituyen como una forma de descentralizar áreas complejas para el manejo administrativo de las IES.

\section{Desarrollo}

Desde sus inicios, la universidad se constituye en la voz y respuesta de las inquietudes del ser humano, y de las sociedades que demandan cambios. En el tiempo el desarrollo de las sociedades ha transformado las funciones de las universidades, por lo que las actividades académicas llevan a los investigadores a trabajar tanto en temas de investigación básica y en innovación comercial, por lo que el reconocimiento laboral ya no es suficiente, si este no logra posicionar a la universidad con el sector productivo.

En Ecuador el modelo de creación de empresas difiere mucho de las dinámicas europeas y norteamericanas, primando el alejamiento hacia los sectores empresariales, debido en muchos casos a las barreras ideológicas respecto de los procesos de vinculación que debieron ser de carácter obligatorios. La persistente lucha por recursos económicos para la investigación, ha venido ocasionando débiles sinergias en los procesos entre los centros de investigación y las universidades, ante lo cual las universidades poco han favorecido la vinculación con empresas privadas o comunidades productivas agrícolas, industriales y comerciales. Por lo que el capital humano no ha logrado sintonizar las necesidades externas de investigación aplicada, desconociendo temas tan elementales como: políticas de propiedad intelectual, patentes, programas de cooperación internacional, financiamiento de fondos concursables para investigación, parques tecnológicos, start ups, incubadoras y spin-offs, etc.

Estas realidades no han sido consideradas en su magnitud, y más bien se dan leyes y procedimientos para crear empresas públicas sin entender adecuadamente los mecanismos que exige el mercado, como bien señalan Jiménez \& Castellanos (2008) al referirse a las limitaciones presentes en los procesos entre universidad y su entorno, consideran que las condiciones socioculturales son adversas a estos procesos y reflejan fuertes separaciones entre la academia y la industria en la región, y la desconfianza por parte de las empresas en relación con colaborar en procesos de investigación con las universidades.

Con lo que se tienen un mecanismo formal que reconoce la necesidad de contar con el respaldo del Consejo Universitario de cada IES como máximo organismo de legislación de la universidad para la creación de una empresa, antes que serios procesos de identificación de necesidades territoriales para su creación y las potencialidades de competir con empresas similares. Casos concretos se pueden observar en la Escuela Superior Politécnica de Chimborazo, que creó la Estación de Servicios ESPOCH Gasolinera Politécnica E.P, con la finalidad de abastecer de combustibles a los vehículos que son propiedad de la institución y el 
público en general. Una actividad que no demuestra una real vinculación con los aspectos anteriormente mencionados de interacción entre universidad y entorno.

Otros casos que se pueden mencionar y que un dista del anterior es el de La Escuela Superior Politecnica del Litoral, la cual crea el 14 de Junio del 2011 con resolución 11-09-169, la Empresa Pública de servicios "ESPOL-TECH”, como una persona jurídica de derecho público, con objeto social más diversificado que la empresa anterior: asesorías, consultorías, control, administración, manejo financiero, gerencia y fiscalización de proyectos, transferencia de tecnologías, cursos de entrenamientos y seminarios; son parte de los servicios que ofrece.

En esta misma línea, el 7 de Noviembre del 2012, mediante Resolución del H. Consejo Directivo en la Universidad de Cuenca se aprueba la creación de la "Empresa Pública de Administración y Gestión de: los servicios, consultoría especializada, y los productos de proyectos de Investigación de la Universidad de Cuenca-UCUENCAEP, como una empresa de personería jurídica de derecho público, con patrimonio propio, autonomía presupuestaria, financiera, económica, administrativa y de gestión en el cumplimiento de su objeto social, fue inscrita en la Notaria Sexta del Cantón Cuenca el dieciséis de Noviembre del 2012.

Con resolución del H. Consejo Universitario N 2260-CU-P-2014, se crea la Empresa Pública de la Universidad Técnica de Ambato UTA-EP. El 8 de noviembre del 2014, en la notaria quinta se crea como una persona jurídica de derecho público, con autonomía presupuestaria, financiera, económica, administrativa y de gestión. Su directorio está integrado por cinco miembros, que salen de los docentes, personal administrativo y de la asociación de cámaras de producción, quienes eligen el gerente general. Su objeto social: administración y gestión de consultoría especializada, los productos de proyectos de investigación, ejecución de obras, prestación de servicios, administración de bienes muebles e inmuebles, operaciones comerciales para vinculación con la universidad y entes externos.

En el año 2016, el H. Consejo Universitario, ratifica la creación y funcionamiento de la empresa pública de bienes y servicios de la Universidad Central con un objeto muy similar a la empresa pública de la universidad de Cuenca y de la ESPOL, lo que lleva a pensar que las IES ven en la consultoría un filón de ingresos extras y una forma de vincularse con el mundo empresarial que demanda servicios e investigaciones especializadas.

Hasta el año 2017, la Secretaría Nacional de Planificación y Desarrollo de Ecuador (SENPLADES en adelante), registra dieciocho empresas públicas pertenecientes a las universidades a nivel nacional, las que tienen se diferencian en su objeto social y en la conformación de sus directorios, en la tabla 1 se hace un recorrido histórico sobre su evolución temporal.

Tabla 1: Relación de empresas públicas ecuatorianas hasta diciembre de 2017

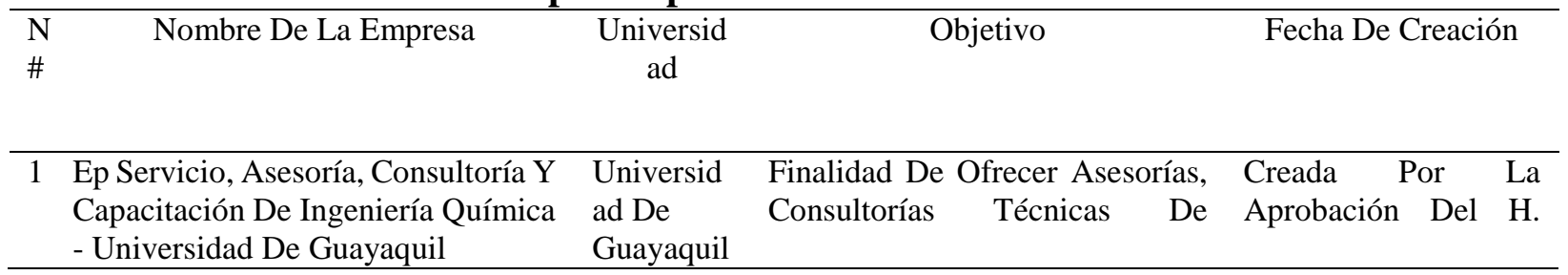




\begin{tabular}{|c|c|c|c|c|}
\hline & & & $\begin{array}{l}\text { Investigación Y Capacitación De } \\
\text { Todo Orden }\end{array}$ & $\begin{array}{l}\text { Consejo Universitario } \\
\text { En El } 2010 .\end{array}$ \\
\hline \multirow[t]{3}{*}{2} & Transespol Ep & Escuela & Prestar Servicio Público De & Creación Resolución \\
\hline & & Superior & Transporte Urbano A Los & $10-03-062$ De 02 \\
\hline & & $\begin{array}{l}\text { Politecnic } \\
\text { a Del } \\
\text { Litoral }\end{array}$ & $\begin{array}{l}\text { Estudiantes, } \quad \text { Profesores, } \\
\text { Empleados Y Funcionarios. }\end{array}$ & Marzo De 2010. \\
\hline 3 & Espolgas Ep & Escuela & Producción, Comercialización, & Resolución \\
\hline \multirow{3}{*}{3} & & Superior & Transporte Y Distribución De & El $\quad 03 \quad$ De \\
\hline & & Politecnic & Combustibles Líquidos, Gas & Diciembre De 2010. \\
\hline & & a Del & Natural Y De Gas Licuado De & \\
\hline & & Litc & Petróleo. & \\
\hline 4 & Hidroespol Ep & Esct & Generación, Compra, Venta De & Resoluciones C.P. 10- \\
\hline 4 & & $\mathrm{Su}$ & Todo Tipo De Energía & 01- $\quad 020 \quad$ De 14 \\
\hline & & $\begin{array}{l}\text { Politecnic } \\
\text { a Del } \\
\text { Litoral }\end{array}$ & $\begin{array}{l}\text { Convencional } \\
\text { Convencional. }\end{array}$ & Diciembre 2010 \\
\hline \multirow[t]{4}{*}{5} & Empresa Pública De Radio, & Escuela & Administración, & Resolución 10-12-367 \\
\hline & Televisión Y Prensa Espol Ep & Superior & Mantenimiento & El 14 De Diciembre \\
\hline & & Politecnic & Radiodifusión, & De 2010. \\
\hline & & $\begin{array}{l}\text { a Del } \\
\text { Litoral }\end{array}$ & Televisión Y Prensa. & \\
\hline \multirow[t]{4}{*}{6} & Escuela De Conductores Profesional & Escuela & Formar Y Adiestrar A Los & Resolución No 11-06- \\
\hline & Espol Ecpe Ep (Conduespol) & $\mathrm{Su}$ & Conductores & 143 Expedida El 07 \\
\hline & & Politecnic & Profesionales Previo A La & Junio De 2011 \\
\hline & & & Obtención De Su Licencia. & \\
\hline \multirow[t]{3}{*}{7} & Empresa Pública Espoltech-Ep. & Escuela & Prestación De Servicios De & Creada Resolución No \\
\hline & & Supe & Asesoría, Consultorías, Control, & 11- 06-169 Expedida \\
\hline & & $\begin{array}{l}\text { Politecnic } \\
\text { a Del } \\
\text { Litoral }\end{array}$ & $\begin{array}{l}\text { Administración De Toda Clase } \\
\text { De Proyectos. }\end{array}$ & $\begin{array}{l}\text { Por El Consejo En } \\
\text { Sesión Del } 14 \text { Junio } \\
\text { De } 2011\end{array}$ \\
\hline \multirow[t]{5}{*}{8} & Empresa Pública De Servicios & Escu & Capacitar A Los Estudiantes De & Creada Resolución No \\
\hline & Educativos Capacita-Espol E.P & Sup & Segundo Nivel Y Bachilleres & 7-219 Expedidas \\
\hline & & Politecnic & Para $\mathrm{Su}$ Ingreso $\mathrm{A}$ Las & El 14 Julio De 2011. \\
\hline & & & Instituciones Del Sistema De & \\
\hline & & Lito & Educación Superior. & \\
\hline \multirow{4}{*}{9} & Empra Pública De Innovación Y & Escuela & Crear Canales Para La & Creada Resolución No \\
\hline & Comercialización & Superior & Comercialización Y Compartir & 11- 07-222 Del 14 \\
\hline & Investigación Inventio-Espol Ep & $\begin{array}{l}\text { Politecnic } \\
\text { a Del }\end{array}$ & $\begin{array}{l}\text { Conocimiento, Tecnología, } \\
\text { Talento Humano, Capacitación Y }\end{array}$ & Julio 2011. \\
\hline & & Lit & Desarrollo De Proyectos. & \\
\hline \multirow{4}{*}{$\begin{array}{l}1 \\
0\end{array}$} & Empresa Pública De Producción Y & Universid & Planificar, Diseñar, Evaluar, & Resolución 63 Del 28 \\
\hline & Desarrollo Estratégico De & & Priorizar Y Ejecutar Los Planes, & De Febrero De 2012. \\
\hline & Universidad Estatal De Quevedo & Tecnica & Programas $\quad \mathrm{Y}$ Proyectos De & \\
\hline & Prodeuteqep & $\begin{array}{l}\text { Estatal De } \\
\text { Quevedo }\end{array}$ & & \\
\hline \multirow{3}{*}{$\begin{array}{l}1 \\
1\end{array}$} & Empresa Pública En La Facultad De & Universid & Servicios De Consultoría De & Resolución No 010- \\
\hline & Ciencias Matemáticas Y Físicas.- Ep & ad De & Ingeniería & 20.06.2012-Ffs-Sg De \\
\hline & $\begin{array}{l}\text { Ingeniería, Materiales Y Sistemas } \\
\text { Ug }\end{array}$ & Guayaquil & $\begin{array}{l}\text { Estructurales, Viales, Hidráulicas } \\
\text { Y Sanitarias. }\end{array}$ & 20 De Junio De 2012. \\
\hline 1 & Empresa Pública Ep Eduquil Ug. & Universid & Producir, $\quad$ Distribuir $\quad \mathrm{Y}$ & Resolución No 011- \\
\hline \multirow[t]{2}{*}{2} & & ad De & Comercializar Publicaciones, Así & 20.06.2012-Ffs-Sg. \\
\hline & & $\mathrm{Gu}$ & $\begin{array}{l}\text { También Hacer Conocer Logros } \\
\text { E Investigaciones. }\end{array}$ & \\
\hline
\end{tabular}




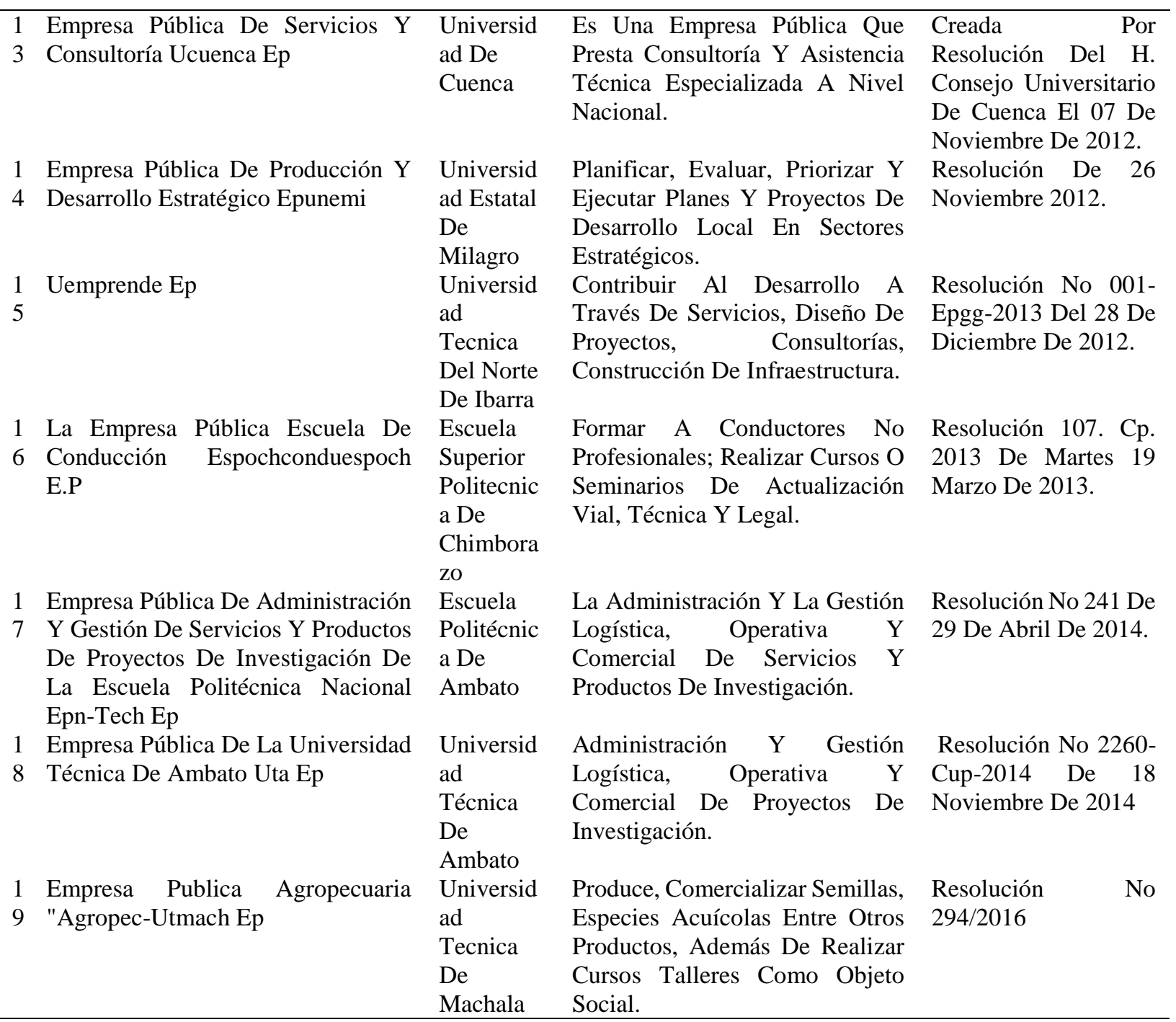

Fuente: (SENPLADES, 2017) (González, 2017) actualizada por los autores

\section{Análisis descriptivo}

\section{Resultados}

En la tabla 2 se evidencia que las empresas públicas creadas por las universidades están orientadas en su mayoría a la prestación de servicios con un $24 \%$, en segundo lugar, se encuentran las relacionadas a los proyectos con un $16 \%$, y posteriormente las consultorías, investigación, comercialización y producción con un $11 \%$. Con menor participación en el mercado están las de formación de conductores con un $5 \%$.

Los resultados obtenidos en la actividad investigativa se ubican por debajo del área de influencia que debe tener una universidad considerando que los objetos sociales no están promoviendo la investigación y la vinculación de sus investigadores con las necesidades de resolver problemas del sector empresarial y comunitario social, al tener objetos muy dispersos como evidencia la tabla 2.

\section{Tabla 2: Objeto Social de las empresas públicas universitarias}




\begin{tabular}{ccc}
\hline Actividades & Universidades & Porcentaje \\
\hline Consultorías & 5 & $14 \%$ \\
Comercialización & 4 & $11 \%$ \\
Administración & 4 & $11 \%$ \\
Producción & 3 & $8 \%$ \\
Prestación de servicios & 9 & $24 \%$ \\
Investigación & 4 & $11 \%$ \\
Proyectos & 6 & $16 \%$ \\
Formación de conductores & 2 & $5 \%$ \\
\hline
\end{tabular}

Fuente: Los Autores

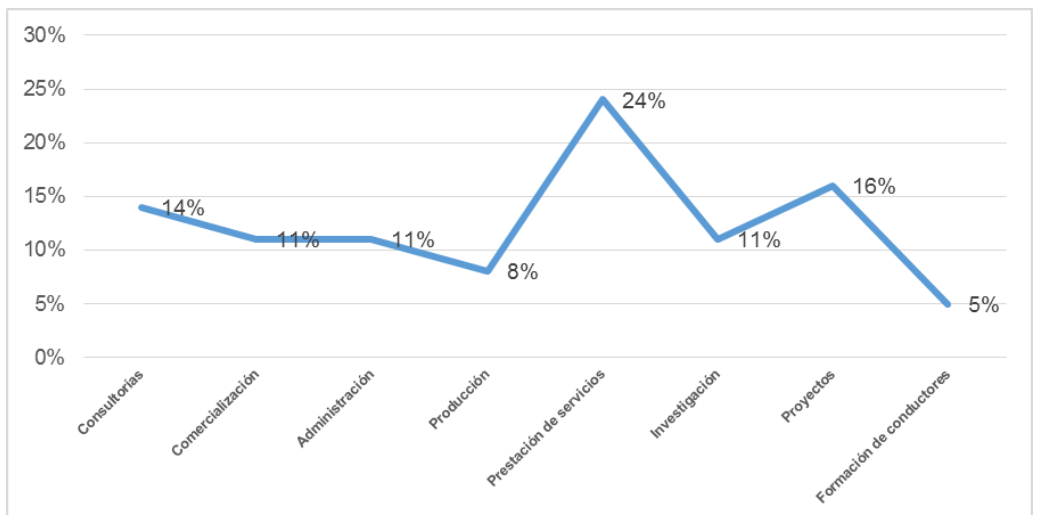

Figura 1. Objeto Social de las empresas públicas universitarias Fuente: Los Autores

Tabla 3: Creación de empresas públicas universitarias

\begin{tabular}{lcc}
\hline \multicolumn{1}{c}{ Universidad } & Número & Porcentaje \\
\hline Universidad De Guayaquil & 3 & $16 \%$ \\
Escuela Superior Politecnica Del Litoral & 8 & $42 \%$ \\
Universidad Tecnica Estatal De Quevedo & 1 & $5 \%$ \\
Universidad De Cuenca & 1 & $5 \%$ \\
Universidad Estatal De Milagro & 1 & $5 \%$ \\
Universidad Tecnica Del Norte De Ibarra & 1 & $5 \%$ \\
Escuela Superior Politecnica De Chimborazo & 1 & $5 \%$ \\
Escuela Politécnica De Ambato & 1 & $5 \%$ \\
Universidad Técnica De Ambato & 1 & $5 \%$ \\
Universidad Tecnica De Machala & 1 & $5 \%$ \\
\hline
\end{tabular}

Fuente: Los Autores

Hay un crecimiento importante de las empresas públicas universitarias, lo que se puede verificar al revisar el trabajo de Miño-Cascante \& Roldan-Ruenes (2015) quienes tomando datos de SENPLADES del 2013 manifiestan la existencia de 10 EP, pasando a 19 empresas públicas en el año 2017, un 90\% de aumento en solo cuatro años, pudiendo pensar que las universidades ven en esta alternativa una buena posibilidad de lograr nuevos ingresos. 


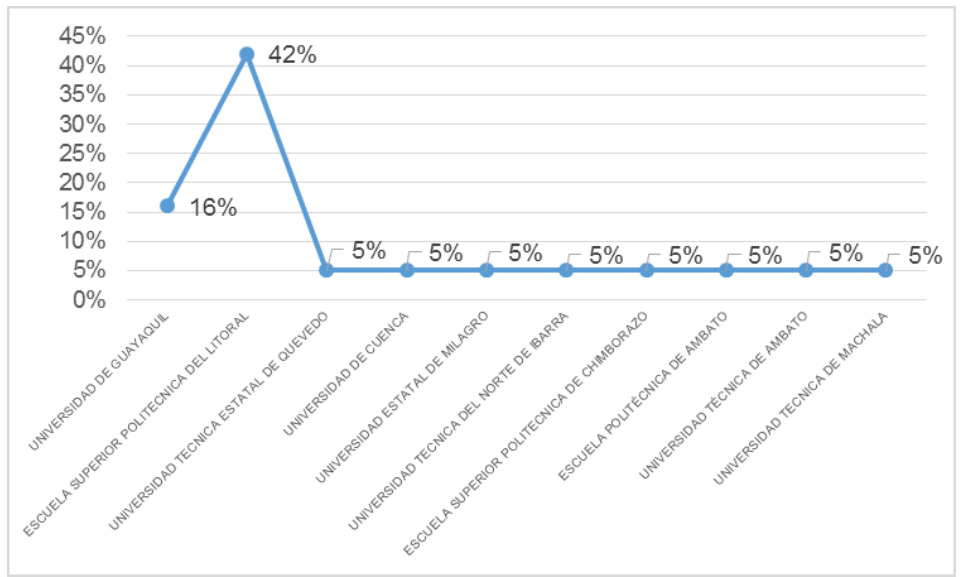

Figura 2. Número de empresas públicas creadas por la IES Fuente: Los Autores

De acuerdo a la figura 2, la universidad que cuenta con mayor número de empresas es Escuela Superior Politécnica del Litoral con un número de 8 que corresponde al 42\%; seguida por la Universidad de Guayaquil con un número de 3 empresas, representando el 16\%; mientras que las universidades Técnica Estatal de Quevedo, Universidad de Cuenca, Universidad Estatal de Milagro, Universidad Técnica del Norte de Ibarra, Escuela Superior Politécnica del Chimborazo; Escuela Politécnica de Ambato; Universidad Técnica de Ambato; Universidad Técnica de Machala, con una empresa cada una que representan por cada universidad el 5\%. Con lo cual, la Escuela Superior Politécnica del Litoral tiene en las EP una forma de acercarse a los proyectos público - privados que demandan contratación de consultorías y prestación de servicios de consultoría, aunque no se conocen como las otras empresas sus resultados y los beneficios alcanzados, así como el grado de cumplimiento de sus objetivos fundacionales.

\section{Leyes conexas para la creación de empresas públicas en el Ecuador}

El marco legal representado en la Constitución de la República del Ecuador artículo. 315, permite que el Estado constituya empresas públicas para la gestión de sectores estratégicos, la prestación de servicios públicos, el aprovechamiento sustentable de recursos naturales o de bienes públicos y el desarrollo de otras actividades económicas. Con lo que las IES aprovechan la ley para mejorar su autonomía financiera, económica, administrativa y de gestión; además, de considerar la Ley Orgánica de Empresas Públicas (LOEP) del año 2009 publicada en el suplemento del Registro Oficial No. 48, y la facultad que confiere de manera puntual al manifestar que: "Las universidades publicas podrán constituir empresas públicas o mixtas que se someterán al régimen establecido en esta Ley para las empresas creadas por los gobiernos autónomos descentralizados o al régimen societario, respectivamente.

En la resolución de creación adoptada por el máximo organismo universitario competente se determinará los aspectos relacionados con su administración y funcionamiento". La denominación de las empresas deberá contener la indicación de "EMPRESA PÚBLICA" o la sigla "EP". En su artículo 39, menciona que para que una empresa nacional pueda ejercer actividades de consultoría, deberá estar constituida de conformidad con la Ley de Compañías y tener en su objeto social incluida esta actividad. 
En el artículo 39, (Ley Orgánica de Educación Superior, 2010) respalda la creación de empresas públicas cuando se realizan actividades económicas, productivas o comerciales, distintas e independientes de la institución educativa, aunque es puntual en no conceder exoneraciones o exenciones tributarias exclusivas a instituciones educativas, ni permite utilizar servicios gratuitos de sus estudiantes, docentes o personal administrativo. De igual forma en el artículo 28, señala que las instituciones de educación superior públicas podrán crear fuentes complementarias de ingresos para mejorar su capacidad académica, invertir en la investigación, en el otorgamiento de becas y ayudas económicas, en formar doctorados, en programas de posgrado, o inversión en infraestructura, en los términos establecidos. Algo que hasta ahora no se conoce muy bien en el sistema de educación superior, al tener poca o nula evidencia del destino de los ingresos de las EP universitarias.

\section{Ley Orgánica del Sistema de Contratación Pública (SNCP)}

Menciona que el servicio Nacional de Contratación Pública tendrá las atribuciones en su artículo 10 en el numeral 8, haciendo mención de promulgar modelos obligatorios de documentos precontractuales y contractuales de los procedimientos para la contratación pública, para ello tendrá el asesoramiento de la Procuraduría General del Estado y de la Contraloría General del Estado, con lo que las empresas públicas no pueden evadir los procedimientos de la contratación pública, con lo que la agilidad que se espera, se pueden pensar que se estanca.

En su artículo 14 numeral 4 de la contratación con proveedores inscritos en el registro único de proveedores (RUP), salvo las excepciones puntualizadas en esta Ley; no representan mayor ayuda para la descentralización de compras de bienes y servicios, por lo que la ley por una parte dice promover la generación de nuevas iniciativas que produzcan recursos a las IES, estas siguen teniendo un comportamiento rígido que es lo que se quiere mejorar, otro aspecto es la revisión de sus actividades por los organismos de control del Estado como la contraloría.

El marco legal que se ha mencionado no tiene un mayor aporte en la gestión organizacional de las EP, que es lo que se busca para poder contratar y ejecutar proyectos de manera más eficiente, pudiendo decir, que no hay mayor aporte en las transacciones que se derivan de las actividades declaradas en las empresas públicas.

\section{Conclusiones}

En el Ecuador, las empresas públicas de las instituciones de educación superior no logran despegar en la generación de nuevos ingresos adicionales que puedan servir para potenciar la investigación y la producción de conocimiento aplicado en su entorno territorial, aunque tienen la obligación legal de participar en el mercado y competir con las empresas privadas, no se puede determinar si el régimen de contratación les facilita poder adquirir bienes y servicios en igualdad de condiciones que las empresas privadas.

En términos específicamente técnicos y a nivel de empresa, no deberían existir diferencias en la eficiencia administración empresarial privada y la pública, sin embargo, no se tiene documentos que demuestren el éxito empresarial de las empresas públicas universitarias, más aún sabiendo que casi todas las universidades públicas tienen facultades de administración de empresas o de ciencias empresariales. 
Las empresas públicas universitarias están fuertemente reguladas y controladas por un gran número de instancias gubernamentales entre las que se pueden mencionar están: Contraloría General del Estado, Instituto de Compras Públicas (INCOP) que está relacionado con el Registro Único de proveedores (RUP) y que obliga a adquirir a proveedores registrados algo que no ocurre con las empresas competidoras, Ley de Finanzas Públicas, Ley de Transparencia, Ministerio de Relaciones Laborales (MRL), Código de Trabajo y LOSEP, Servicio de Rentas Internas (SRI), Instituto Ecuatoriano de Seguridad Social (IESS), Ministerio de Finanzas, Banco Central del Ecuador, y otras específicas de los sectores donde se desarrollen sus actividades como Ministerio del Ambiente, Ministerio de Hidrocarburos, entre otras. Con lo que hay un excesivo control burocrático que resta competitividad con las empresas privadas que ofrecen servicios semejantes.

El acceso al financiamiento privado es otro de los obstáculos que impide que estas propuestas empresariales puedan escalar en tamaño, teniendo que depender de los pocos recursos que se inyecten desde sus creadores o de los contratos relacionados que se presenten. Además, de no tener un grupo de indicadores de gestión que las orienten en la consecución de sus fines declarados en sus actos constitutivos. No hay procesos de rendición de cuentas a los dueños de las empresas públicas, ni tampoco se sabe de informes que manifiesten el uso de las utilidades obtenidas anualmente, y la participación de empresas en concursos nacionales públicos y privados.

\section{Bibliografía}

Beraza, J., \& Rodriguez, A. (2010). Los programas de apoyo a la creacion de Spin-offs en las universidades Españolas: una comparacion internacional. Investigaciones Europeas de Dirección y Economía de la Empresa, 17(2), 89-117. Recuperado el 8 de 12 de 2017, de http://redaedem.org/articulos/iedee/v17/172089.pdf

Constitución de la Republica del Ecuador, Asamblea Constituyente 2008. Art. 226. (2008). Recuperado el 22 de 07 de 2017, de http://www.derechoambiental.org/Derecho/Legislacion/Constitucion_Asamblea_Ecuador_4.html

Constitucion de la Republica del Ecuador, Asamblea Constituyente 2008. Art. 315. (s.f.). Recuperado el 22 de 08 de 2018, de http://www.derechoambiental.org/Derecho/Legislacion/Constitucion_Asamblea_Ecuador_4.html

Escuela Superior Politécnica del Chimborazo. (29 de 11 de 1983). Recuperado el 21 de 08 de 2017, de https://www.espoch.edu.ec/index.php/gasolinera-politecnica.html

Escuela Superior Politecnica del Litoral. (14 de 06 de 2011). Recuperado el 21 de 07 de 2017, de http://www.espol-tech.espol.edu.ec/sites/default/files/CREACION\%20ESPOL\%20TECH.pdf

Ferreiro, F. J. (2016). Análisis de los viveros de empresas en Galicia según su orientación tecnológica o generalista. Cuadernos de Gestión, 16(2), 49-76. Recuperado el 25 de 07 de 2017, de www.ehu.eus/cuadernosdegestion/documentos/130451ff.pdf

Fong, C. (2010). Transferencia de conocimiento de la Universidad a la empresa: La creación de empresas Spin-off de origen universitario. Sistemas, Cibernetica e Informática, 7(1), 81-88. Recuperado el 10 de 12 de 2017, de http://www.iiisci.org/journal/CV\$/risci/pdfs/MJ639IY.pdf 
Gibson, D. V., \& Mahdjoubi, D. (2010). Diferentes aspectos de la Transferencia construyendo una red de transferencia de tecnologia competitiva a nivel global. Revista Galega de Economía, 19, 112. Recuperado el 10 de 12 de $2017, \quad$ de http://www.usc.es/econo/RGE/Vol19_ex/castelan/art12c.pdf

González, F. (2017). Las Empresas Públicas en el Ecuador: su situación jurídica y su régimen laboral. Quito: Universidad de Cuenca.

Iglesias, P., Jambrino, C., \& Peñafiel, A. (2012). Caracterización de las Spin-off universitarias como mecanismo de transferencia de tecnología a través de un análisis clúster. Revista Europea de Dirección y Economia de la Empresa., 21(3), 240-254.

Jiménez, C., \& Castellanos, O. (2008). Desafios en gestón ecnológica para las universidades. Congreso Internacional de Gestión Tecnológica e Innovación., (págs. Jiménez, C. y Castellanos, O.). Bogotá.

Ley Orgánica de Educación Superior. (12 de 10 de 2010). Recuperado el 22 de 08 de 2017, de file://C:/Users/Trabajo/Downloads/loes\%20(1).pdf

Leydesdorff, L., \& Etzkowitz, H. (1998). The Triple Helix as a model for innovation studies. Science and Public Policy, 25(3), 195-203.

Lopez, P. (2017). Surgimiento de empresas catalogadas como spin-off universitarias en Colombia, análisis desde la gerencia de proyectos (fase I). Escuela de Administración de Negocios(82), 61-72. Recuperado el 10 de 12 de 2017, de http://www.redalyc.org/pdf/206/20652069008.pdf

Méndez, M., Hernández, J., Lino, J., \& González, M. (2014). Estudio Exploratorio de las Spin Off desde la perspectiva de los gestores Universitarios. European Scientific Journal, 10(16), 380391. Recuperado el 10 de 07 de $2017, \quad$ de eujournal.org/index.php/esj/article/download/3534/3306

Miño-Cascante, G., \& Roldan-Ruenes, A. (2015). La gestión de empresas públicas universitarias en el Ecuador. Anuario, Facultad de Ciencias Económicas y empresariales, 3-20.

SENPLADES. (2017). Empresa pública y planificación. Su rol en la transformación social y productiva 2017.

Suplemento del Registro Oficial N 48. Ley Orgánica de Empresas Públicas. (16 de 10 de 2009). $\begin{array}{lllllll}\text { Recuperado el } & 22 & \text { de } & 08 & \text { de } & 2017, & \text { de }\end{array}$ https://www.google.com.ec/search?q=ley+organica+de+empresas+publicas+art.+5\&oq=ley+ organica+de+empresas+publicas+art. $+5 \&$ gs_l=psy-

ab.3...3876.8056.0.8371.9.9.0.0.0.0.444.1480.21j0j3.4.0.foo\%2Cers1\%3D1\%2Cfett\%3D1\%2Cewh\%3D0\%2Cnso-enksa\%3D0\%2Cnsoenfk\%3D0.3.

Universidad de Cuenca. (07 de 11 de 2012). Recuperado el 21 de 08 de 2017, de http://ucuencaep.com.ec/quienes-somos/historia/ 